\title{
ALCOHOL ABUSE ENHANCES SYSTEMIC INFLAMMATORY RESPONSE IN PATIENTS AFTER SPONTANEOUS INTRACEREBRAL HAEMORRHAGE
}

\author{
M. Danovska ${ }^{1}$, M. Alexandrova ${ }^{2}$, D. Peychinska ${ }^{1}$, I. Gencheva ${ }^{3}$ \\ 1) Neurology Clinic, 3) Medical Diagnostic Clinical laboratory, University \\ Hospital Pleven, Bulgaria \\ 2) Department of Physics and Biophysics, Medical University Pleven, Bulgaria
}

\section{SUMMARY}

OBJECTIVE: The role of inflammation in the complex pathophysiology of spontaneous intracerebral hemorrhage (sICH) was studied by assessing the relationship between serum C-reactive protein (CRP) levels and some clinical and neuroradiological parameters. We also aimed to identify the effects of modifiable vascular risk factors on serum CRP levels.

PATIENTS: Forty six patients with sICH admitted to the Department of Neurology and Neurosurgery of the Pleven University Hospital, Bulgaria were examined. Serum CRP levels were measured within the first 48 hours of disease onset and analyzed in relation to neurological deficit severity and clinical outcome after sICH. The impact of some vascular risk factors on the inflammatory marker levels was also studied.

RESULTS: We found enhanced CRP levels in patients with severe neurological deficit as assessed by the National Institutes of Health Stroke Scale (NIHSS) score. Significantly higher CRP levels were measured in patients with progressive clinical deterioration and worse outcome. Serum CRP levels were also higher in patients with a history of alcohol abuse.

CONCLUSIONS: Our results suggest that inflammation plays a crucial role in the development of brain injury after sICH. They show that CRP, a nonspecific inflammatory marker, can serve as an additional diagnostic and prognostic test indicator in the acute stage of sICH thus providing an excellent opportunity for therapeutic interventions while the patient is still in clinic. Patients with a history of systemic alcohol abuse demonstrate stronger inflammatory response indicative for worse prognosis.

Key words: Intracerebral hemorrhage, inflammation, CRP, alcohol abuse, arterial hypertension.

\section{INTRODUCTION}

Spontaneous intracerebral hemorrhage (sICH) is a devastating type of stroke characterized with severe disability and the highest rate of mortality ${ }^{(1)}$. It accounts for $15-20 \%$ of all strokes ${ }^{(2)}$. Despite the outstanding achievements in the modern diagnosis of sICH, the outcome after ICH has not been improved - its treatment is still symptomatic and the results achieved are very unsatisfactory ${ }^{(3,4)}$. The cerebral hemorrhage is considered to initiate a complex cascade of metabolic events causing neuronal damage and cellular death ${ }^{(5,6,7)}$. The inflammation plays a central role in the molecular mechanisms triggered after the hemorrhage onset ${ }^{(8)}$. There is data published on the role of C-reactive protein (CRP), an acute phase reactant, in the pathogenesis and prognosis of acute ischemic stroke ${ }^{(8)}$. Moreover increased CRP levels also indicate higher risk of future cerebrovascular events ${ }^{(9,10)}$. But the role of CRP in the pathogenesis of cerebral hemorrhage remains unresolved yet. It is not clear whether CRP only shows systemic inflammation, or is directly involved in the mechanisms of brain injury after sICH.

Obesity, alcohol consumption and smoking have been identified as risk factors for vascular events. They also play an essential role in the etiology and pathogenesis of atherosclerosis and arterial hypertension, which are considered the major causes for stroke ${ }^{(11,12)}$. There are a few scientific publications confirming that initiation of inflammatory reactions in the vascular endothelium causes cerebrovascular disorders ${ }^{(13)}$. CRP has been detected in atherosclerotic plaques and probably contributes to atherogenesis and initiates pro-coagulation state $(13,14)$. Furthermore, CRP levels are also correlated with arterial hypertension ${ }^{(12)}$. Evidence has been published showing that some risk factors as smoking, alcohol consumption and hyperglycemia cause increased CRP serum levels $\left.{ }^{(15,16}\right)$. As CRP is frequently associated with cerebrovascular diseases and their prognosis it will be useful to investigate the relationship between CRP and the neurologic deficit severity after ICH in the context of risk factors for stroke.

The purpose of the present study was:

1. To investigate the relationship between serum CRP level and stroke severity and outcome in patients after acute 
ICH.

2. To search for additive effect of concomitant risk factors implementation on CRP levels in patients after ICH.

\section{MATERIALAND METHODS}

We examined 46 patients ( 23 men and 23 women), mean age of $63 \pm 2$ years, who suffered a moderate to severe intracerebral hemorrhage and were admitted to the Department of Neurology and Neurosurgery, University Hospital, Pleven, Bulgaria. Intracranial hemorrhage was diagnosed through neurological examination and CT scan of the brain. Blood cell count, biochemical parameters and serum CRP levels were measured within the first 48 hours of the hemorrhage onset. The patients and their relatives responded to a special questionnaire asking for a range of personal, demographic and medical data. Through the questionnaire we also obtained detailed information on the patient's history of vascular risk factors, coexisting diseases and family illnesses. Stroke severity was assessed by Glasgow Coma Scale (GCS), disability by NIHSS and functional outcome at discharge by Glasgow Outcome Scale (GOS) and modified Rankin Scale (mRS). Patients with clinical symptoms of acute infectious disease, history of malignancy, chronic kidney and liver diseases were excluded from the study. All patients had the consent form signed either by themselves or on their behalf by a close relative.

\section{Statistical analysis}

The statistical analysis was performed using Statistical Package for Social Sciences, version 12.0 (SPSS Inc., Chicago, IL). The interval variables were defined as medial value (standard deviation) or median (25-75 percentile) based on the type of data distribution. The normal distribution was determined by Shapiro - Wilk test. The significance of the differences among the groups was defined by Mann-Whitney test and Kruskal Wallis. [Spearman's correlative analysis was performed to evaluate the relationship between the clinical variables and the CRP serum level]. For the purpose of assessing the relationship between the clinical variables and CRP serum level a correlative analysis of Spearman was performed. The value of $p<0.05$ was set as a limit for statistical significance.

\section{RESULTS}

The basic characteristics of the patients examined are shown in Table 1.

Higher serum CRP levels were found in patients with severe neurological deficit or disability assessed by NIHSS (Spearman correlation: $R=0.384, p=0.009)$ (Fig 1.), and in patients with poor functional outcome at discharge, assessed by GOS (Spearman correlation $R=-0.374$, $P=0.011$ ) (Fig.2) and mRS (Spearman correlation $R=0.386$, $p=0.008$ ) (Fig.3). Moreover, patients that showed progressive clinical deterioration had higher serum levels of the inflammatory marker as compared to patients that presented gradual improvement of the neurological state (Kruskal Wallis test, $u=8.629 ; p=0.013$ ) (Fig.4). Patients with a history of alcohol abuse had higher serum CRP levels as compared to those without a history of alcohol abuse (Mann- Whitney U test, $p=0.027$ ) (Fig.5).

\section{DISCUSSION}

Acute intracerebral hemorrhage is due to spontaneous bleeding in the brain parenchyma. Currently this is the most devastating type of stroke with serious medical, social and economic consequences. The lack of effective ICH therapy increases the necessity for better understanding of the complex pathophysiological mechanisms that initiate cellular necrosis and apoptosis after ICH. Moreover, a better knowing of some essential modifiable risk could provide an additional chance for modeling and controlling the disease course with final positive impact on prognosis of the sICH.

There is growing experimental and clinical evidence that confirms the key role of inflammation not only in the pathogenesis of atherosclerosis, but also for the increased incidence of cerebrovascular and cardiovascular events ${ }^{(8,}$ 10). Although a nonspecific inflammatory marker, the acute phase reactant CRP, has earned popularity in the assessment of inflammation ${ }^{(10)}$. Yan et al ${ }^{(17)}$ found significantly higher CRP levels in patients with intracerebral hemorrhage and these levels correlated with worse clinical outcome. Our results also demonstrated higher CRP levels in patients with poor functional outcome and clinical deterioration who were assessed by GOS and mRS. This data gave us substantial evidence to hypothesize that CRP could be a useful marker in predicting ICH prognosis.

Arterial hypertension is the most studied risk factor for $\mathrm{ICH}^{(6)}$. Relatively unexplored are other risk factors that are closely related to the etiopathogenesis of $\mathrm{ICH}^{(2,11)}$. This fact could be explained with the lack of enough objective data necessary for the risk assessment and its real significance for the course and prognosis of ICH. A very interesting relationship between alcohol consumption (data collected from the interview) and CRP level was found after risk factors analysis was performed in patients with ICH. Significantly higher were found the CRP levels in patients with a history of alcohol abuse $(p=0.027)$. A positive correlation between alcohol consumption and the risk of ICH was also shown in some data published $(15,16,18,19,20)$. There are studies that do not accept the role of alcohol intake as a risk factor for the development of $\mathrm{ICH}^{(21)}$. According to Saloheimo ${ }^{(21)}$ only recent heavy drinking, but not a history of heavy drinking, was a risk factor for ICH in patients at middle and advance age. Leppdld et al. ${ }^{(22)}$ found linear dose response relationship between the alcohol intake and the risk for ICH.

It is considered that alcohol consumption increases 
the risk of $\mathrm{ICH}$ basically by its relationship with arterial hypertension. But alcohol also decreases platelet aggregation and causes coagulation disorders (23). An increased fibrinolytic activity and decreased fibrinogen levels, predisposing for prolonged bleeding were found in patients with alcohol abuse. Theoretically alcohol enhances vascular fragility ${ }^{(11)}$. The possibility to identify chronic alcohol abuse as a risk factor for $\mathrm{ICH}$ is of great importance because it could offer a unique chance to influence the frequency and the course of ICH. A better understanding of the additive effect exerted by alcohol on inflammation could also improve our knowledge in ICH pathogenesis. Taking into consideration the results published a reasonable explanation of the correlation between systemic alcohol consumption and serum CRP levels could be found in the direct alcohol effects upon coagulation status and blood vessels integrity. Probably our patients with $\mathrm{ICH}$ had prolonged bleeding that induced a stronger local inflammatory response and caused higher serum CRP level

Table 1. Baseline characteristics of study population

\begin{tabular}{|l|r|}
\hline PARAMETER & \\
\hline Age [years], (SD) & $63(2)$ \\
\hline Male sex, $\mathrm{n}(\%)$ & $23(50.0)$ \\
\hline Arterial hypertension, $\mathrm{n}(\%)$ & $40(87)$ \\
\hline Systolic blood pressure [mm Hg], (SD) & $186(35)$ \\
\hline Diastolic blood pressure [mm Hg], (SD) & $103(18)$ \\
\hline Ichemic heart disease, $\mathrm{n}(\%)$ & $10(22)$ \\
\hline Diabetes mellitus, $\mathrm{n}(\%)$ & $4(9)$ \\
\hline Alcohol abuse, $\mathrm{n}(\%)$ & $28(61)$ \\
\hline Cigarette smoking, $\mathrm{n}(\%)$ & $17(40)$ \\
\hline GOS score at discharge, median $\left(25^{\text {th }}-75^{\text {th }}\right)$ & $3(1-4)$ \\
\hline NIHSS score on day 7, median $\left(25^{\text {th }}-75^{\text {th }}\right)$ & $19(16-25)$ \\
\hline WBC $\left[x 10^{9} / 1\right],(\mathrm{SD})$ & $10.2(3.5)$ \\
\hline Glucose [mmol/1], median $\left(25^{\text {th }}-75^{\text {th }}\right)$ & $6.6(5.5-8.1)$ \\
\hline Total cholesterol [mmol/1], $(\mathrm{SD})$ & $5.90(1.32)$ \\
\hline Fibrinogen $[\mathrm{g} / 1],(\mathrm{SD})$ & $3.85(1.17)$ \\
\hline
\end{tabular}

being the major indicator for the stronger systemic inflammatory response.

Despite the long history of poor clinical outcome and high mortality, optimism exists that $\mathrm{ICH}$ management could be improved for the future on the base of new insights into the acute pathophysiology of ICH. A better understanding of the dynamic processes of hematoma enlargement, the importance of inflammation, triggered by the coagulation and the products of blood degradation, the harmful effects of hyperthermia and hyperglycemia may provide additional optional targets for future interventions in the complex management of ICH ${ }^{(24)}$. The development of novel antiinflammatory agents, targeting the coagulation-induced brain injury, respectively thrombin, hemoglobin, Matrix Metalloproteinases (MMP) end endothelial growing factors, may change the historical concept for $\mathrm{ICH}$ been the most devastating illness in the clinical practice into a treatable condition worthy of an aggressive and promising approach.

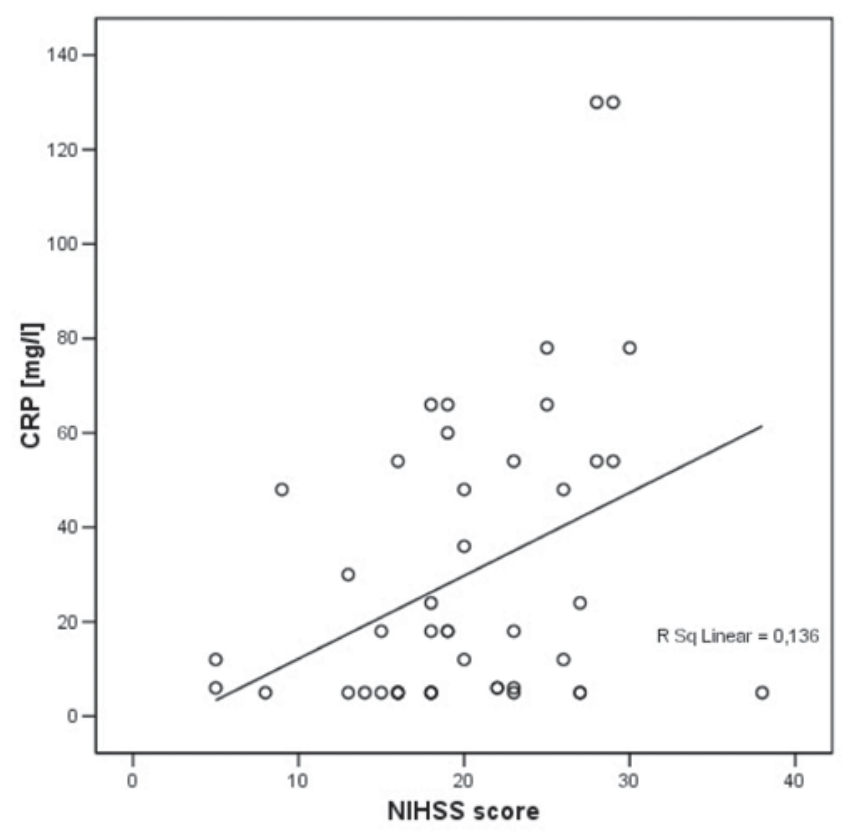

Fig. 1. Correlative significance between the serum CRP levels and neurologic deficit severity, assessed by NIHSS (Spearman correlation) 


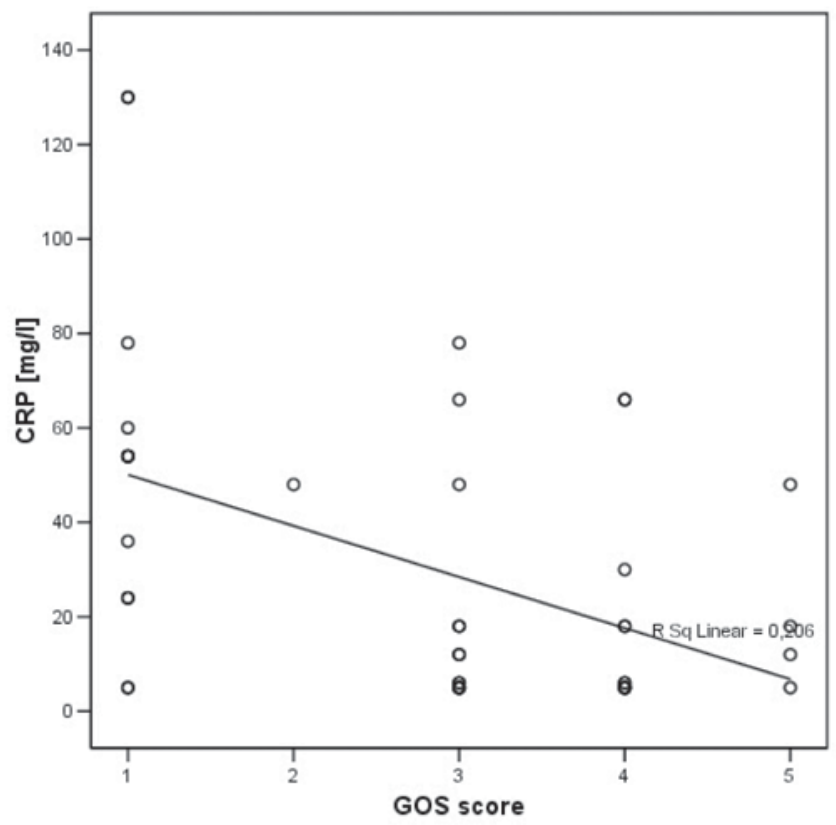

Fig. 2. Correlative dependence between the serum CRP levels and functional outcome assessed by GOS (Spearman correlation)

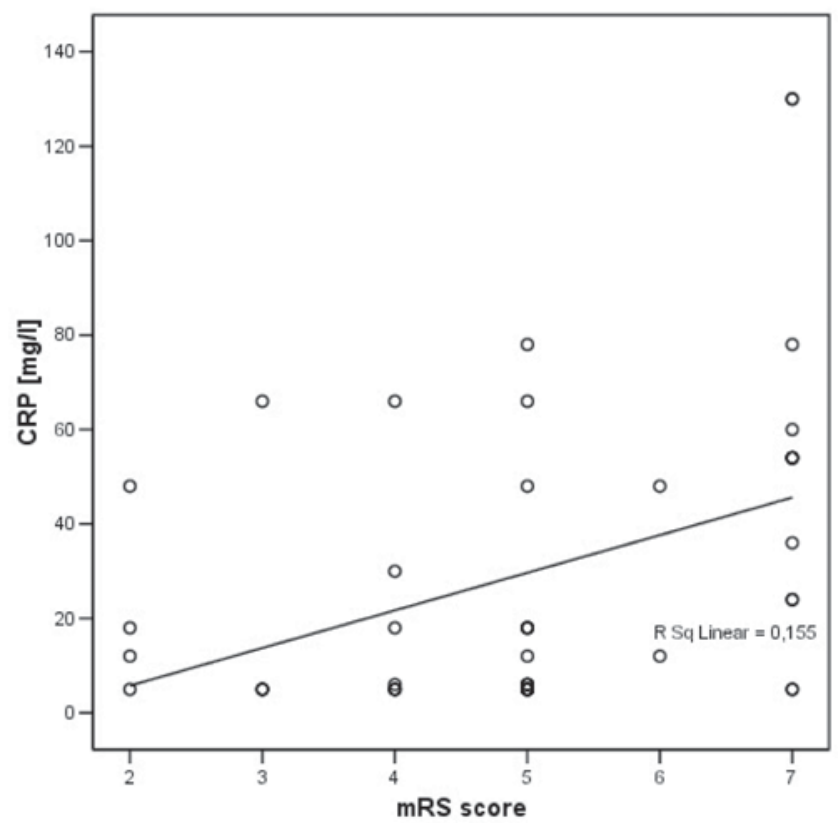

Fig. 3. Correlative dependence between the serum CRP levels and functional outcome assessed by mRS (Spearman correlation)

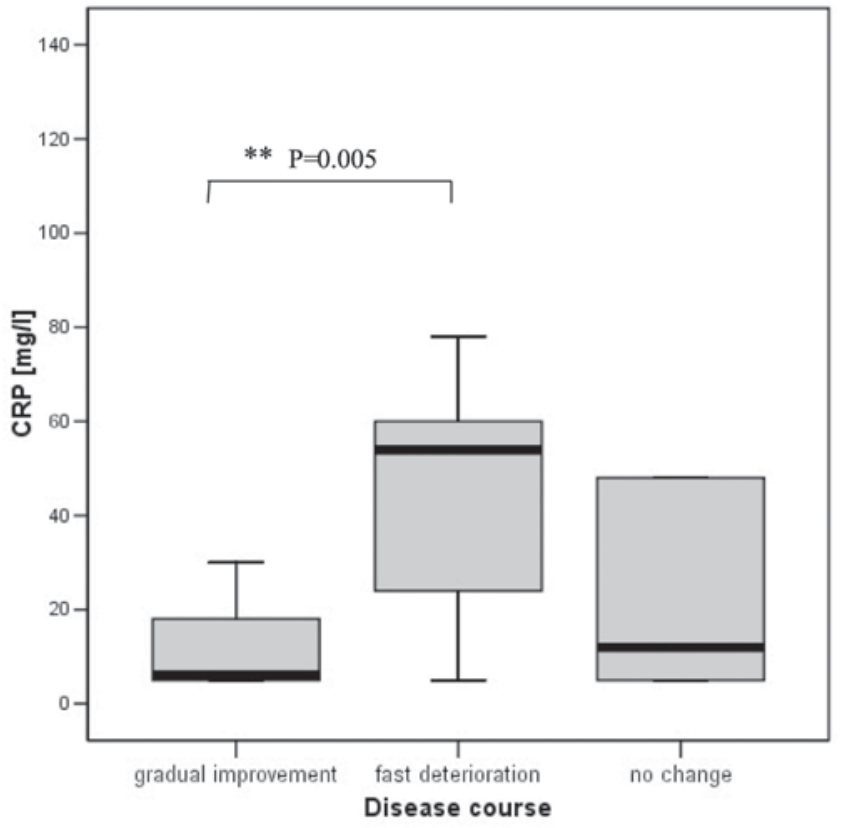

Fig. 4. Serum CRP levels in relation with the disease course (Kruskal Wallis test)

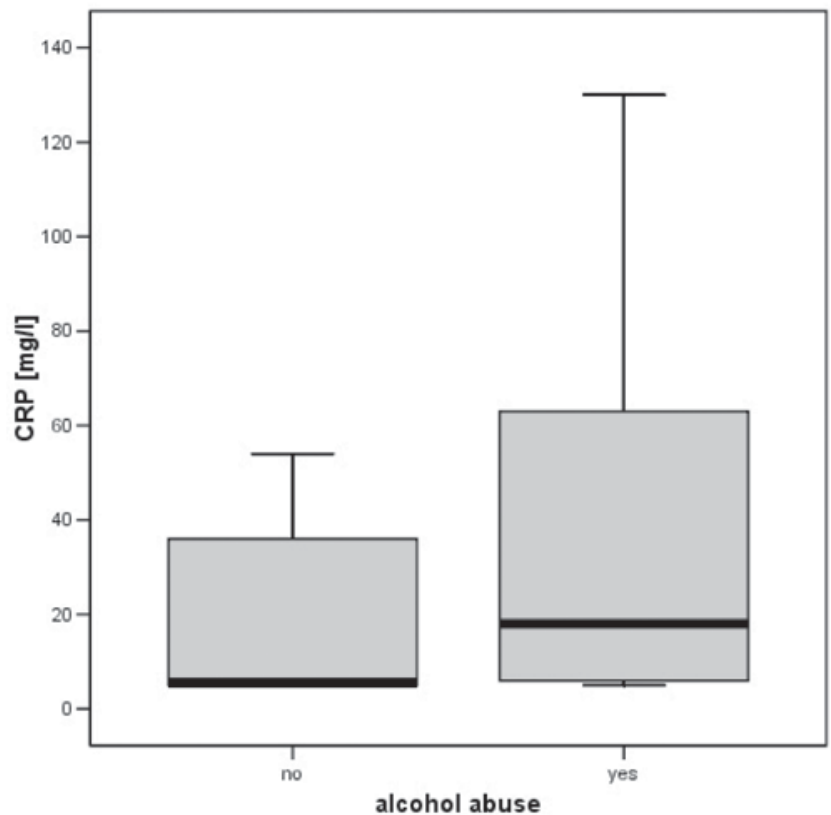

Fig. 5. Serum CRP levels in patients with $\mathrm{ICH}$ and chronic alcohol abuse (Mann-Whitney U test) 


\section{REFERENCES:}

1. Broderick J, Conolly S, Feldmann E, Hanley D, Kase C, Krieger D, et al. American Heart Association; American Stroke Association Stroke Council; High Blood Pressure Research Council; Quality of Care and Outcomes in Research Interdisciplinary Working Group: Guidelines for the management of spontaneous intracerebral hemorrhage in adults: 2007 update: a guideline from the American Heart Association/American Stroke Association Stroke Council, High Blood Pressure Research Council, and the Quality of Care and Outcomes in Research Interdisciplinary Working Group. Stroke 2007; 38:20012023.

2. Qureshi, A, Mendelow, D, Hanley, D. Intracerebral hemorrhage. Lancet, 2009, 373, 1632-44.

3. Ribo M, Grotta J. Latest advances in intracerebral hemorrhage. Curr Neurol Neurosci, 2006, 6, 17-22.

4. Rincon F, Mayer S. Clinical review; Critical care management of spontaneous intracerebral hemorrhage. Critical care 2008; $12 ; 237$.

5. Carmichael S, Vespa P, Saver J, Coppola G, Geschwind D, Starkman S, et al. Genomic profiles of damage and protection in human intracerebral hemorrhage. J Cereb Blood Flow Metab. 2008; 28(11):1860-1875.

6. Xi G, Keep R, Hoff J. Mechanisms of brain injury after intracerebral hemorrhage. Lancet Neurol, 2006, 5, 53-63.

7. Zhang L, Liu C, Zhu C, Liu R, Li C. Brain edema after intracerebral hemorrhage in rats: the role of inflammation Neurol India 2006; 54:402.

8. Wang J, Dore S. Inflammation after intracerebral hemorrhage. J Cerebr Blood F Met 2007 894-908.

9. Alexandrova, M, Bochev, P. Oxidative stress in stroke. Oxidative stress and neurodegenerative disorders, 2007 Elsevier, 307-362.

10. Di Napoli, M., Arakelyan, A., Boyajiyan, A., Godoy, D., Papa, F. The acute phase inflammatory response in stroke: systemic inflammation and neuroinflammation. Progress in Inflammation Research, 2006, Nova Science Publishers Inc, 95-145.

11. Herzig R, Urbanek K, Vlachova I, Krupka B, Gabrys M, Mares J, et al. The role of chronic alcohol intake in spontaneous intracranial hemorrhage: a carbohydratedeficient transferring study. Cerebrovasc Dis 2003; 15:22-28.

12. Xu T, Ju Z, Tong W, Hu W, Liu Y, Zhao L et al. Zhang Y, Relationship of CReactive Protein With Hypertension and Interactions Between Increased C-Reactive Protein and Other Risk Factors on Hypertension in Mongolian People, China. Circ J 2008; 72: 1324-1328

13. Schwartz RS, Bayes-Genis A, Lesser JR, Sangiorgi M, Henry TD, Conover CA. Detecting vulnerable plaque using peripheral blood: Inflammatory and cellular markers. J Interv Cardiol 2003; 16 : 231-242.

14. Torzewski J, Torzewski M, Bowyer DE, Frohlich M, Koenig W, Waltenberger J, et al. C-reactive protein frequently colocalizes with the terminal complement complex in the intima of early atherosclerotic lesions of human coronary arteries. Arterioscler Thromb Vasc Biol 1998; 18: 1386- 1392.
15. Thrift A, Donnan G, McNeil J. Heavy drinking, but not moderate or intermediate drinking, increases the risk of intarcerebral hemorrhage. Epidemilogy 1999; 30:537-541.

16. Zodpey S, Tiwari R, Kulkarni H. Risk factors for hemorrhagic stroke: a casecontrol study. Public Health 2000; 114:177182.

17. Yan J, Hui R, Wang D. Elevated Creactive protein levels predict worsening prognosis in Chinese patients with firstonset stroke. Front Med China 2009, 3 (1):30-35.

18. Gorelick P. Alcohol and stroke. Stroke 1988; 19:48-52.

19. Juvela S, Hillbom M, Palomaki H. Risk factors for spontaneous intracerebral hemorrhage Stroke 1995;26:1558-1564.

20. Klatsky A, Armstrong M, Friedman G. Risk of cardiovascular mortality in alcohol drinkers, ex-drinkers and nondrinkers. Am J cardiol.1990; 66:1237-1242.

21. Saloheimo P, Juvela S, Hillbom M. Use of aspirine, epistaxis, and untreated hypertension as risk factors for primary intracerebral hemorrhage in middle-aged and elderly people. Stroke 2001; 32:399-404.

22. Leppala J, Paunio M, Virtamo J, Fogelholm R, Albanes D, Taylor P, et al. Alcohol consumption and stroke incidence in male smokers. Circulation 1999; 100: $1209-214$.

23. Hillbom M. Oxidants, antioxidants, alcohol and stroke. 1999 Frontiers and Bioscience 4 67-71.

24. Kleinig, T, Vink, R. Suppression of inflammation in ischemic and hemorrhagic stroke: therapeutic options. Curr Opin Neurol, 2009, 22, 294-301.

\author{
Address for correspondence: \\ Maya Danovska, MD, \\ Second Neurology Clinic, University Hospital Pleven, Bulgaria \\ 8A George Kochev Str., 5800 Pleven, Bulgaria \\ Tel: +35964886276, \\ E-mail: mdanovska@yahoo.com
}

